\title{
Prediction of the Impact of the Nozzle Geometry on Spray \\ Characteristics
}

Mahmoud Abd Elaziz Mohamed*, Hesham Elsayed Abdel Hameed, Ramy Elsayed Shaltout, and Hafez Abdel Aal El-Salmawy

Department of Mechanical Power Engineering, Faculty of Engineering, Zagazig University, Sharkia 4419, Egypt

*Address for correspondence:

Mahmoud Abd El-Aziz Mohamed

Department of Mechanical Power Engineering

Faculty of Engineering,

Zagazig University, Sharkia 4419, Egypt;

Email: eng.mah1981@yahoo.com 


\section{Appendices}

\section{$\underline{\text { Appendix-A }}$}

$C_{k}$ and $C_{\mathcal{E}}$ are coefficient which determined dynamically by the test-scale. This scale is constructed from the grid-scale by applying a test filter which is characterized by $\left[(\Delta)_{f}=2 \bar{\Delta}\right]$, where the subscribt symbol $(f)$ is employed for the test filtering. $C_{k}$ and $C_{\mathcal{E}}$ are defined as following ${ }^{1}$.

$C_{k}=\frac{1}{2} \frac{L_{i j} \sigma_{i j}}{\sigma_{i j} \sigma_{i j}}$,

$L_{i j}$ is Leonard stress tensor is defined as:

$L_{i j}=\left(\overline{u_{i}} \overline{u_{j}}\right)_{f}-\left(\overline{u_{i}}\right)_{f}\left(\overline{u_{j}}\right)_{f}$

And $\sigma_{i j}=-(\Delta)_{f} k_{t e s t}^{1 / 2}\left(\bar{S}_{i j}\right)_{f}$

On the other hand:

$C_{\varepsilon}=\frac{\left[\left\langle\left(\frac{\partial \bar{u}_{i}}{\partial x_{j}}\right)\left(\frac{\partial u_{i}}{\partial x_{j}}\right)\right\rangle_{f}-\left\langle\frac{\partial\left(\bar{u}_{i}\right)_{f}}{\partial x_{j}}\right\rangle\left\langle\frac{\partial\left(\bar{u}_{i}\right)_{f}}{\partial x_{j}}\right\rangle\right]}{\left.\left(\mu+\mu_{t}\right)(\Delta)_{f}\right]^{-1} k_{\text {test }}^{3 / 2}}$

Where: $k_{\text {test }}=\frac{1}{2}\left[\left(\bar{u}_{k} \bar{u}_{k}\right)_{f}-\left(\bar{u}_{k}\right)_{f}\left(\bar{u}_{k}\right)_{f}\right]$ 


\section{$\underline{\text { Appendix-B }}$}

The drag coefficient equations used by Morsi and Alexander ${ }^{2}$ are:

$$
\begin{aligned}
& C_{D}=\frac{24}{\operatorname{Re}_{r}} \\
& C_{D}=\frac{22.73}{\operatorname{Re}_{r}}+\frac{0.0903}{\operatorname{Re}_{r}{ }^{2}}+3.69 \quad \text { for } 0.1<\mathrm{Re}_{r}<1.0 \\
& C_{D}=\frac{29.1667}{\operatorname{Re}_{r}}-\frac{3.8889}{\operatorname{Re}_{r}{ }^{2}}+1.222 \quad \text { for } 1.0<\mathrm{Re}_{r}<10.0 \\
& C_{D}=\frac{46.5}{\operatorname{Re}_{r}}-\frac{116.67}{\operatorname{Re}_{r}{ }^{2}}+0.6167 \quad \text { for } 10.0<\mathrm{Re}_{r}<100.0 \\
& C_{D}=\frac{98.33}{\operatorname{Re}_{r}}-\frac{2778}{\operatorname{Re}_{r}{ }^{2}}+3.644 \quad \text { for } 100.0<\mathrm{Re}_{r}<1000.0 \\
& C_{D}=\frac{148.62}{\operatorname{Re}_{r}}-\frac{4.75 \times 10^{4}}{\operatorname{Re}_{r}{ }^{2}}+0.357 \quad \text { for } 1000.0<\operatorname{Re}_{r}<5000.0 \text {, } \\
& C_{D}=\frac{-490.546}{\operatorname{Re}_{r}}+\frac{57.87 \times 10^{4}}{\operatorname{Re}_{r}{ }^{2}}+0.46 \quad \text { for } 5000.0<\operatorname{Re}_{r}<10000.0, \\
& C_{D}=\frac{-1662.5}{\operatorname{Re}_{r}}+\frac{5.4167 \times 10^{6}}{\operatorname{Re}_{r}{ }^{2}}+0.5191 \text { for } 10000.0<\operatorname{Re}_{r}<50000.0 .
\end{aligned}
$$

\section{REFERENCES}

(1) Kim, W.-W.; Menon, S.; Kim, W.-W.; Menon, S. Application of the Localized Dynamic Subgrid-Scale Model to Turbulent Wall-Bounded Flows. In 35th aerospace sciences meeting and exhibit; 1997; p 210.

(2) Morsi, S. A. J.; Alexander, A. J. An Investigation of Particle Trajectories in Two-Phase Flow Systems. J. Fluid Mech. 1972, 55 (2), 193-208. 